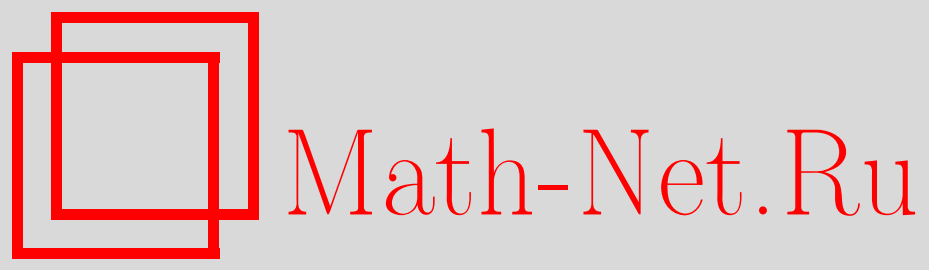

М. И. Кадец, Об абсолютной, совершенной и безусловной сходимости двойных рядов, Функи. анализ и его прил., 1996, том 30, выпуск 2, 85-86

DOI: https://doi.org/10.4213/faa527

Использование Общероссийского математического портала MathNet.Ru подразумевает, что вы прочитали и согласны с пользовательским соглашением

http://www . mathnet.ru/rus/agreement

Параметры загрузки:

IP : 3.89 .185 .249

26 апреля 2023 г., 09:53:38

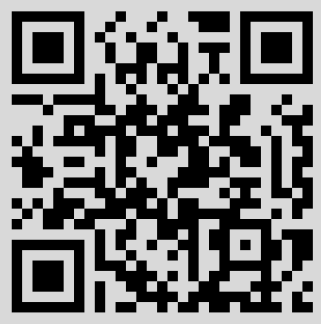


УДК 517.52

\title{
Об абсолютной, совершенной и безусловной сходимости двойных рядов
}

\author{
(C) 1996. М. И. КАДЕЦ ${ }^{1}$
}

Ряд $\sum x_{n}$ в пространстве Банаха $X$ называется абсолютно сходящимся, если сходится числовой ряд $\sum\left\|x_{n}\right\|$. Ряд называется совершенно сходящимся, если для любого набора коэффициентов $\alpha_{n}= \pm 1$ (обозначение: $\alpha \in D$ ) сходится ряд $\sum \alpha_{n} x_{n}$. Ряд называется безусловно сходящимся, если для любой перестановки натурального ряда $\pi: \mathbb{N} \rightarrow \mathbb{N}$ сходится ряд $\sum x_{\pi(n)}$. Понятия совершенной и безусловной сходимости эквивалентны. Каждый абсолютно сходящийся ряд сходится совершенно. Обратное верно в том и только в том случае, когда пространство конечномерно. Для дальнейшего нам потребуется банахово пространство $\operatorname{Perf}(X)$ всех совершенно сходящихся рядов в $X$. Норма в этом пространстве определяется как $\sup \left\{\left\|\sum \alpha_{n} x_{n}\right\|: \alpha \in D\right\}$. По поводу упомянутых понятий и фактов см., например, монографию [1].

Перейдем к рассмотрению двойных рядов. Прежде всего дадим определение сходимости двойного ряда, являющееся некоторым усилением определения сходимости по Принсгейму.

ОПРЕДЕЛЕНИЕ 1 . Ряд $\sum x_{i j}$ в банаховом пространстве $X$ сходится к сумме $s \in X$, если (а) сходится каждый «ряд-столбец» $\sum_{i} x_{i j}, j \in \mathbb{N}$, (b) сходится каждый «ряд-строка» $\sum_{j} x_{i j}, i \in \mathbb{N}$, (c) сходится последовательность "прямоугольных» частных сумм:

$$
s=\lim s_{m n}=\lim \left\{\sum_{i=1}^{m} \sum_{j=1}^{n} x_{i j}: \min (m, n) \rightarrow \infty\right\} .
$$

Понятия абсолютной, совершенной и безусловной сходимости для двойных рядов, хорошо согласованные со структурой этих рядов, можно ввести следующим образом.

ОПРЕДЕЛЕНИЕ 2. Ряд $\sum x_{i j}$ называется абсолютно сходящимся, если сходится числовой ряд $\sum\left\|x_{i j}\right\|$.

ОПРЕДЕЛЕНИЕ 3. Ряд $\sum x_{i j}$ называется совершенно сходящимся, если для любых наборов коэффициентов $\alpha, \beta \in D$ сходится ряд $\sum x_{i j} \alpha_{i} \beta_{j}$.

ОПРЕДЕЛЕНИЕ 4. Ряд $\sum x_{i j}$ называется безусловно сходяшимся, если для любых перестановок натурального ряда $\pi$ и $\sigma$ сходится ряд $\sum x_{\pi(i), \sigma(j)}$.

Следующее утверждение характеризует совершенную сходимость в терминах линейных операторов.

ТЕорема 1. Для двойного рлда $\sum x_{i j}$ в банаховом пространстве $X$ следующие условия эквивалентнь: (А) рлд сходится совершенно, (В) вектор-

\footnotetext{
${ }^{1}$ Результаты настоящей заметки получены при поддержке Международного научного фонда, грант №V9H000.
} 
нозначная матрича $A=\left(x_{i j}\right)$ порождает линейный компактный оператор $A: c_{0} \rightarrow \operatorname{Perf}(X)$, действуюший по правилу $A e_{j}=\left\{x_{i j}\right\}_{i=1}^{\infty}, j \in \mathbb{N}$, где $\left(e_{j}\right)$ - канонический базис пространства $c_{0}$. Норма этого оператора равна

$$
M(A)=\sup \left\{\left\|\sum x_{i j} \alpha_{i} \beta_{j}\right\|: \alpha, \beta \in D\right\} .
$$

Две другие теоремы описывают связь между совершенной, безусловной и абсолютной сходимостью двойных рядов.

Теорема 2. Двойной ряд сходится совершенно в том и только том случае, если он сходится безусловно.

ТЕОрема 3. Существуют двойные числовые ряды, сходящиеся совершенно, но не абсолютно.

ЭСКИЗ докАЗАТЕльСТвА. Требуемый пример мы построим из «блоков»отнормированных соответствующим образом матриц Уолша. Напомним, что матрицей Уолша $W_{n}, n \in \mathbb{N}$, называется ортогональная матрица порядка $m=2^{n}$, все элементы которой равны \pm 1 . Последовательность матриц Уолша можно определить по индукции:

$$
W_{1}=\left(\begin{array}{cc}
1 & 1 \\
1 & -1
\end{array}\right), \quad W_{2}=\left(\begin{array}{cc}
W_{1} & W_{1} \\
W_{1} & -W_{1}
\end{array}\right) \text { и т.д. }
$$

Сумма абсолютных величин элементов матрицы Уолша равна $N\left(W_{n}\right)=m^{2}$. Функционал (1) допускает оценку $M\left(W_{n}\right) \leqslant m^{3 / 2}$. Введем теперь матрицы $A_{n}=m^{-2} W_{n}$. Ясно, что $N\left(A_{n}\right)=1$ и $M\left(A_{n}\right) \leqslant 1 / \sqrt{m}$. Образуем бесконечную матрицу $A=\left(a_{i j}\right)$, поставив на ее диагонали матрицы $A_{n}$ и положив все остальные элементы равными нулю. Двойной ряд, порожденный этой матрицей, сходится совершенно $(M(A) \leqslant \sqrt{2}+1)$, но не абсолютно $(N(A)=\infty)$.

ЗАмечание 1 . Теорема 1 и теорема 3 остаются верными, если в определении 1 отбросить условия (a) и (b). Однако теорема 2 при этом окажется неверной. Соответствующий пример - двойной ряд $\sum a_{i j}$, где

$$
a_{1 j}=(-1)^{j}, a_{2 j}=-a_{1 j}, a_{i j}=0 \quad \text { при } i>2, j \in \mathbb{N} .
$$

ЗАМЕЧАНИЕ 2. Несовпадение безусловной и абсолютной сходимости для числовых двойных рядов отчасти «компенсируется» следующим утверждением: если ряд $\sum a_{i j}$ безусловно сходится, то сходится ряд $\sum a_{i j}^{2}$.

\section{ЛитературА}

1. Kadets V. M., Kadets M. I. Rearrangements of Series in Banach Spaces. Transl. Math. Monographs, Vol. 86, Am. Math. Soc., 1991.

Харьковская государственная академия Поступило в редакцию городского хозяйства 26 августа 1994 г. 